Cell Research (2000), 10, 151-160

\title{
Leafy head formation of the progenies of transgenic plants of Chinese cabbage with exogenous auxin genes
}

\author{
He Yu Ke*, Wan Xin Xue, Yu Dong Sun, Xu Hong Yu, \\ Ping Lin LiU \\ National Laboratory of Plant Molecular Genetics, Shanghai \\ Institute of Plant Physiology, Chinese Academy of Sciences, \\ Shanghai 200032, China
}

\begin{abstract}
The experiment was performed to evaluate the progenies of plant lines transgenic for auxin synthesis genes derived from Ri T-DNA. Four lines of the transgenic plants were self-crossed and the foreign auxin genes in plants of T5 generation were confirmed by Southern hybridization. Two lines, D1232 and D1653, showed earlier folding of expanding leaves than untransformed line and therefore had early initiation of leafy head. Leaf cuttings derived from plant of transgenic line D1653 produced more adventitious roots than the control whereas the cuttings from folding leaves had much more roots than rosette leaves at folding stage, and the cuttings from head leaves had more roots than rosette leaves at heading stage. It is demonstrated that early folding of transgenic leaf may be caused by the relatively higher concentration of auxin. These plant lines with auxin transgenes can be used for the study of hormonal regulation in differentiation and development of plant organs and for the breeding of new variety with rapid growth trait.
\end{abstract}

Key words: Auxin genes, Chinese cabbage, leafy head, transgenic plants

\section{INTRODUCTION}

Chinese cabbage ( Brassica campestris ssp.pekinensis) is one of the most important

* Corresponding author. Fax:021-64042090; E-mail: heyk@iris. sipp.ac.cn 
Auxin genes and Leafy head

vegetable crops in China. Leaf head, the storage organ for Chinese cabbage, is composed of a number of heading leaves which usually initiate after rosette stage. The rosette leaves differentiate at rosette stage and form what is commonly referred to as a frame which functioned as photosynthesis organ during plant growth, while the heading leaves surrounding the shoot apexes are tight enough to form heads or hearts and thus become storage organ for essential nutrients. Usually, the production of Chinese cabbage is hampered by poor heading of leaves. Many factors such as temperature, light intensity, auxin concentration and ratio of carbohydrate to nitrogen are considered to be closely related to the formation of leaf head[1]. Among them, endogenous auxin is the most important component in that its concentration and uneven distribution highly could modify the processes of leaf bending and folding[2]. Although treatment of leaves with auxin (IAA or NAA) appears to influence the formation of leafy head[3], difficulties and laborious work involved in such treatment limit the study of auxin function in leaf head formation. In 1994, we introduced a set of auxin synthesis genes (auxin gene) into Chinese cabbage and mainly evaluated the root development in the case of their elevated concentration and uneven distribution[4]. The auxin genes employed were isolated from Ri T-DNA in pRIA4 plasmid of wild type of Agrobacterium rhizogenes strain A4[5]. The set of auxin genes is composed of two auxin genes ( aux1 and aux2) and an intergenic promoter sequence. aux1 and aux 2 respectively encode tryptophan monooxygenase and indoleacetamide hydrolase, the enzymes needed for two-step synthesis of IAA[6],[7]. The cloning of auxin genes makes it possible to analyze the function of auxin genes in formation of leafy head.

After transgenic lines of Chinese cabbage were obtained and evaluated, we made genetic analysis by the self-cross and sib-cross of different lines and eventually got the pure lines of transgenic plants in the fifth generation. Within population of each line, genetic segregation between individual plants was not recognized. In this report, plant lines transgenic for auxin genes are examined with regard to leaf phenotype and heading.

\section{MATERIALS AND METHODS}

\section{Plant material}

The TT505 of Chinese cabbage ( Brassica campestris ssp.pekinensis) was employed. The method for transformation of plants with auxin genes and identification of transgenic lines were described previously[4]. The shoots and plants directly regenerated from transformed roots are regarded as those of $\mathrm{T} 0$ generation while $\mathrm{T} 5$ represents the fifth generation of the transgenic plants after self-pollination. Seeds of T5 generation were selected carefully and sowed in greenhouse on August 10 of 1998, and the seedlings were transplanted respectively in greenhouses and in open field four weeks after sowing. Since TT505 was self-incompatible and could not fertilize normally with self-pollens when flower was opened, the flower buds of transgenic lines and untransformed line (control) were emasculated and manually pollinated 1-4 d before flower buds opened. Fully matured seeds were collected, stored at $4{ }^{\circ} \mathrm{C}$ for two weeks.

\section{Plant growth analysis}

The seeds of transgenic lines were sown aseptically on MS solid medium and seed germination 
performance was investigated. In the open field, growth parameters were recorded as to the time of folding and heading, number of rosette leaves at folding stage and head phenotype for evaluation.

\section{Biological assays of auxin}

Leaf samples were collected respectively from folding leaves at folding stage and leaf cuttings were made by cutting leaf in size of $1 \mathrm{~cm}^{2}$ and put aseptically on MS solid medium with or without $0.5 \mathrm{mg} / \mathrm{L}$ IAA. Rooting time and number of adventitious roots on each leaf cutting were recorded.

\section{Southern hybridization}

Two plants of each transgenic line were selected for Southern hybridization. Total DNA was extracted respectively from leaf of each plant and digested with EcoRI and transferred onto nitrocellulose membrane and prehybridized for $4 \mathrm{~h}$ at $42{ }^{\circ} \mathrm{C}$ in prehybridization solution containing $25 \%$ formamide, $100 \mu \mathrm{g} / \mathrm{ml}$ denatured salmon sperm DNA, $10 \mathrm{mg} / \mathrm{ml}$ yeast RNA, $5 \times$ Denhardt' $\mathrm{s}$ solution, $50 \mathrm{mM}$ sodium phosphate $(\mathrm{pH} \mathrm{6.5),} 5 \times \mathrm{SSC}$ and $0.2 \%$ SDS. The $6.1 \mathrm{~kb}$ fragment of AUX genes containing two IAA synthesis genes was cut with SalI from pBluescript plasmid and labled with ${ }^{32} \mathrm{P}$ dCTP using random primer method[8]. The resultant DNA probe was added to the prehybridized solution and hybridized respectively with plant DNA. The hybridized filter was rinsed in leaching solution containing $0.05 \mathrm{M} \mathrm{NaH}_{2} \mathrm{PO}_{4}, 0.05 \mathrm{M} \mathrm{NaHPO} 4,5 \times \mathrm{SSC}$ and $25 \%$ formamide at room temperature, and once in $2 \times \mathrm{SSC} / 0.02 \% \mathrm{SDS}$ at $42 \mathrm{oC}$. The filters were dried and exposed to x-ray film with intensifying screens overnight.

\section{RESULTS}

Originally, auxin synthesis genes derived from Ri T-DNA can incite the formation of adventitious roots on the explants infected with Agrobacterium. In the previous study, induction and growth of adventitious roots initiated by exogenous auxin genes were analyzed extensively in Brassica crops, including cabbage, Chinese cabbage and cauliflower. Individual transformed roots induced on cotyledon sections cocultured with Agrobacterium grew into different transformed root lines after several times of subcultures and careful selection on the MS medium containing kanamycin[4]. According to root length and number of branch roots, four types of root lines were classified: longer and more numerous; longer and less numerous; shorter and more numerous; shorter and less numerous. The plants (T0 generation) regenerated from root segments of these root lines were sorted into three groups on the basis of plant phenotype: D1232 with a slightly abnormal shoot and fast growth; D1645 with extremely abnormal shoot and inhibitory growth, and D1434 with normal shoot like the control. It was found that longer and numerous roots was correlated with the fast grown shoots, shorter roots with inhibitory shoots, and longer and less numerous roots with normal shoots. Thus a correlation between root growth and plant phenotype of $\mathrm{T} 0$ generation was established.

In the present study, the focus was put on the properties of leaf folding and leafy head formation of progenies of Chinese cabbage with auxin transgenes. In greenhouses, transgenic plants of $\mathrm{T} 5$ generation or untransformed plants grew well, but failed to produce the normal leafy heads at last and therefore were not assayed in the following 
Auxin genes and Leafy head

experiments. The plants in open field grew normally, produced leafy heads and were investigated further.

Among four transgenic lines (T5 generation) of Chinese cabbage examined, three lines, D1232, D1470 and D1653 were modified with plant leaf and growth performance, whereas the other line, D1911, produced plant phenotype similar to that of untransformed line. During vegetative growth of transgenic plants, seed germination, time of beginning and duration of developmental stages, leaf folding and head formation were recorded, and then the function of the exogenous auxin genes in transgenic plants was analyzed.

\section{Seed germination of transgenic lines}

Seeds of four transgenic lines and one untransformed line were selected and used for germination test. The seeds of most plant lines germinated normally and grew up to seedlings like normal seeds (Tab 1). D1232 and D1653 had nearly the same germination percentage as control. Most seeds of D1470 germinated abnormally as they formed either radicals without cotyledon or abnormal cotyledon without radicals. The resultant plants did not grow well and stopped growing at one of the developmental stages, and were therefore abandoned at last. Three seeds germinated normally were examined further. Among three transgenic lines, the abnormally germinated seeds of D1470 were much more than D1232 or D1653. On the contrary, the seeds of either D1911 or control germinated normally.

Tab 1. Seed germination of transgenic lines of Chinese cabbage (T5 generation). 50 seeds per line were used for seed germination.

\begin{tabular}{lccccc}
\hline Seed performance & \multicolumn{5}{c}{ Transgenic lines } \\
\hline \multirow{3}{*}{ Seed number } & D1232 & D1470 & D1653 & D1911 & Control \\
Germinated & 50 & 50 & 50 & 60 & 60 \\
Germinated abnormally & 43 & 3 & 45 & 50 & 49 \\
No germination & 6 & 37 & 4 & 0 & 0 \\
\hline
\end{tabular}

\section{Leaf folding}

About $50 \mathrm{~d}$ after sowing, plants had grown up to 25 basal leaves (rosette leaves) approximately, while the folding leaves that oriented upward and inward began to differentiate at the sites of shoot apex. The first developing leaf that oriented inward might be considered as the most outer folding leaf, which could be taken as a morphological marker of leaf folding. Thus, the date when $50 \%$ plants from one population of each line had the first folding leaf was designated as the beginning of leaf folding. According to this standard, the plants of D1232 and D1653 lines started leaf folding on September 22nd, one week earlier than normal plants (control) (Tab 2). Among four transgenic lines, D1653 and D1232 grew faster. The sign of leaf folding was seen in plants of D1653 as early as six weeks after sowing. 
He YK et al.

Tab 2. Leaf folding of several plant lines of Chinese cabbage with auxin transgenes.

\begin{tabular}{|c|c|c|c|}
\hline Plant lines & $\begin{array}{c}\text { Folding time } \\
\text { (days after sowing) }\end{array}$ & $\begin{array}{r}\text { Number of rosette les } \\
(60 \mathrm{~d} \text { after sowing }\end{array}$ & $\begin{array}{l}\text { Number of total leaves } \\
\text { (60 d after sowing) }\end{array}$ \\
\hline D1232 & 42 & $23 \pm 2.8$ & $44 \pm 1.2$ \\
\hline D1470 & $\mathrm{n}$ & $26 \pm 2.1$ & $26 \pm 1.9$ \\
\hline D1653 & 42 & $23 \pm 1.5$ & $43 \pm 1.5$ \\
\hline D1911 & 49 & $28 \pm 1.1$ & $39 \pm 2.4$ \\
\hline Control & 49 & $28 \pm 2.9$ & $38 \pm 3.6$ \\
\hline
\end{tabular}

Rosette leaves and total leaves of longer than $5 \mathrm{~mm}$ were counted. The date when $50 \%$ plants from one population of each line had the first folding leaf was designated as the beginning of leaf folding. All data except that of folding time, measured $60 \mathrm{~d}$ after sowing, are the mean of 20 plants and are shown \pm standard experiment error. $\mathrm{n}$, not determined.

Number of rosette leaves and total leaves was determined two months after sowing. At early heading stage, plant of D1232 or D1653 had rosette leaves five less but total leaves five or six more than that of control. Since total leaves included folding leaves and head leaves, decrease in number of rosette leaves means more folding and head leaves in D1232 and D1653. D1470 was a special transgenic line, whose leaves were severely wrinkled and grew abnormally, and all became rosette leave.

\section{Leafy head formation}

The characters of leafy head formation were investigated. The D1232 and D1653 plants grew very rapidly, and each plant gave rise to hard head of full size (Tab 3). In contrast, the D1470 plants grew very slowly and abnormally, and failed to form any normal head.

Introduction of auxin genes did not change the head shape. The TT505 of Chinese cabbage has round-shaped heads. T5 plants, both transgenic and non-transgenic, also produced round-shaped heads. Compared with leafy heads of control, the heads of D1232 and D1653 plants were heavier and consisted of more head leaves. However, there was no significat difference in head sizes between different lines.

Tab 3. Characters of leaf head formation of $\mathrm{T} 5$ plants from Chinese cabbage with auxin transgenes.

\begin{tabular}{|c|c|c|c|}
\hline \multirow[t]{2}{*}{ Plant lines } & \multicolumn{3}{|c|}{ Head characters } \\
\hline & Head weight $(\mathrm{kg})$ & Head height $(\mathrm{cm})$ & Leaf number per head \\
\hline$\overline{\mathrm{D} 1232}$ & $1.8 \pm 0.6$ & $25.5 \pm 1.2$ & $53 \pm 1.2$ \\
\hline D1653 & $1.9 \pm 0.2$ & $25.8 \pm 0.6$ & $54 \pm 0.8$ \\
\hline D1911 & $1.6 \pm 0.8$ & $25.6 \pm 0.9$ & $48 \pm 0.9$ \\
\hline Control & $1.6 \pm 0.4$ & $25.5 \pm 1.7$ & $48 \pm 1.4$ \\
\hline
\end{tabular}

All data, measured $110 \mathrm{~d}$ after sowing, are the mean of 20 plants and are shown

\pm standard experiment error.

\section{Root initiation on leaf cuttings}

From plants of transgenic line D1653 at folding stage, folding leaves (developing leaves) were cut into sections and then intact leaf (IL), intact blade (without petiole) (IB) and apical blade halves $(\mathrm{ABH})$ were transferred on MS medium without hormone. Adven- 
Auxin genes and Leafy head

titious roots began to proliferate on cut ends of the leaf sections in about 14 days after transfer. All the three leaf sections produced roots while the most roots were identified on cut ends of petiole in intact leaf. However, in this case, there was not any root differentiated either on intact blade or on apical blade halves of control plant, although a small number of short adventitious roots were seen on intact leaf of control plant (Fig 1A).
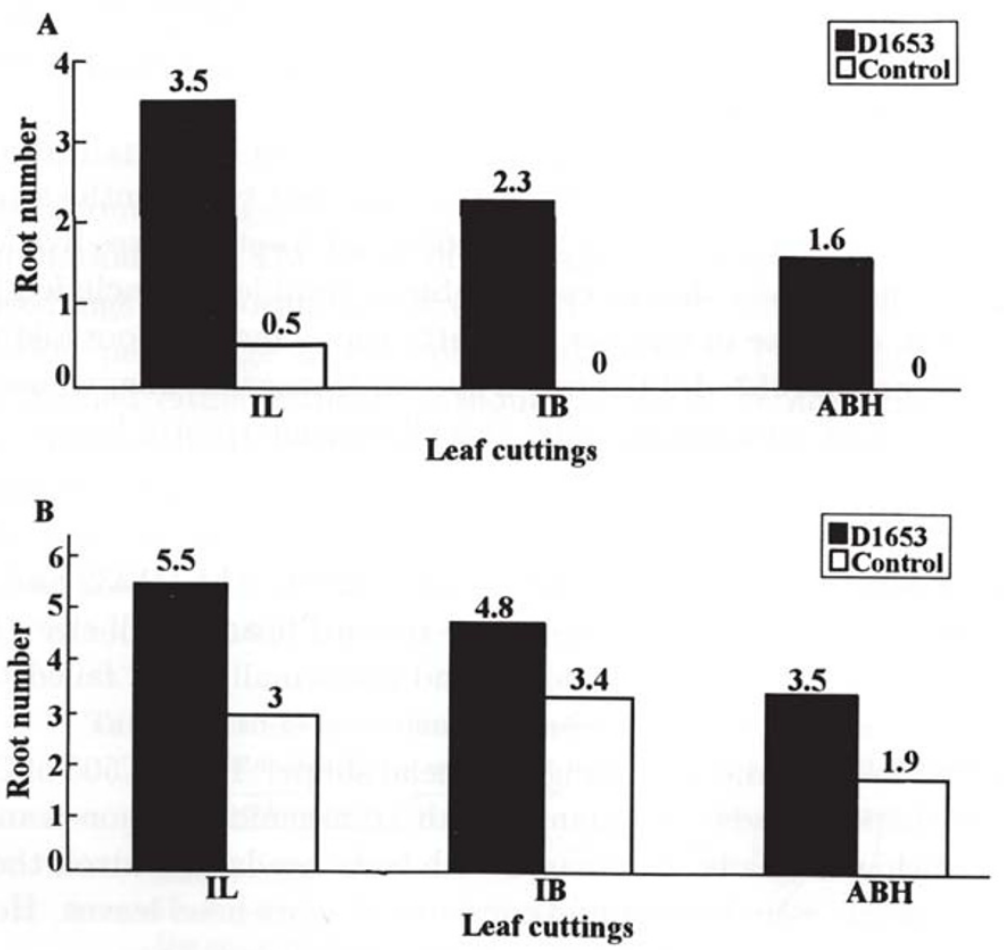

Fig 1.

Number of adventitious roots on leaf cuttings of plants of Chinese cabbage carrying auxin transgenes on MS medium without auxin (A) and with IAA (B). All data, measured $25 \mathrm{~d}$ after initial culture, are the mean of 20 leaves or leaf sections. $\mathrm{ABH}$, apical blade halves; IB, intact blades; IL, intact leaves.

On MS medium containing $0.5 \mathrm{mg} / \mathrm{L}$ IAA, both transgenic sections and control sections produced adventitious roots. Apparently, much more roots were seen on transgenic leaf sections (Fig 1B).

\section{Identification of foreign auxin genes in transgenic plants}

The total DNA was extracted from leaves of T5 transgenic plants and Southern hybridization was performed to identify the foreign auxin genes. There was no hybridization signal with the untransformed plants (the control), demonstrating that AUX genes 
He YK et al.

derived from Ri T-DNA of Agrobacterium rhizogenes was not homologous to plant auxin synthesis genes in Chinese cabbage. All four transgenic lines showed hybridization signal although the hybridization band of D1653 was faint (Fig 2). Originally, the insert of auxin genes was $6 \mathrm{~kb}$ in length and had one EcoRI site $1.2 \mathrm{~kb}$ downstream from ATG condon of aux1. When total DNA of transgenic plant was digested with EcoRI, two hybridization bands could be predicted in the case of one copy of auxin genes. However, three bands were recognized with transgenic lines D1232 and D1470 and only one band with D1653 and D1911. Maybe there were other bands with D1653 or D1911 that was too faint to observe. These data are not sufficient to determine the copy number of auxin genes in transgenic plants. Thus, more experiments need to be carried out to evaluate the gene integration.

Fig 2.

Southern blots of auxin synthesis gene aux in transgenic Chinese cabbage. Genomic DNA was digested with EcoRI and electrophoresed in $0.8 \%$ agarose gel. The DNA was transferred onto nitrocellulose membrane and hybridized with the probe of auxin gene ( aux1 and aux2) labeled with ${ }^{32} \mathrm{P}$ by random priming. Auxin gene marker of $6.0 \mathrm{~kb}$ is in lane 1, and D1232, D1470, D1653, D1911 and untransformed lines are respectively in lanes $2,3,4,5,6$. The DNA size is shown on the left side.

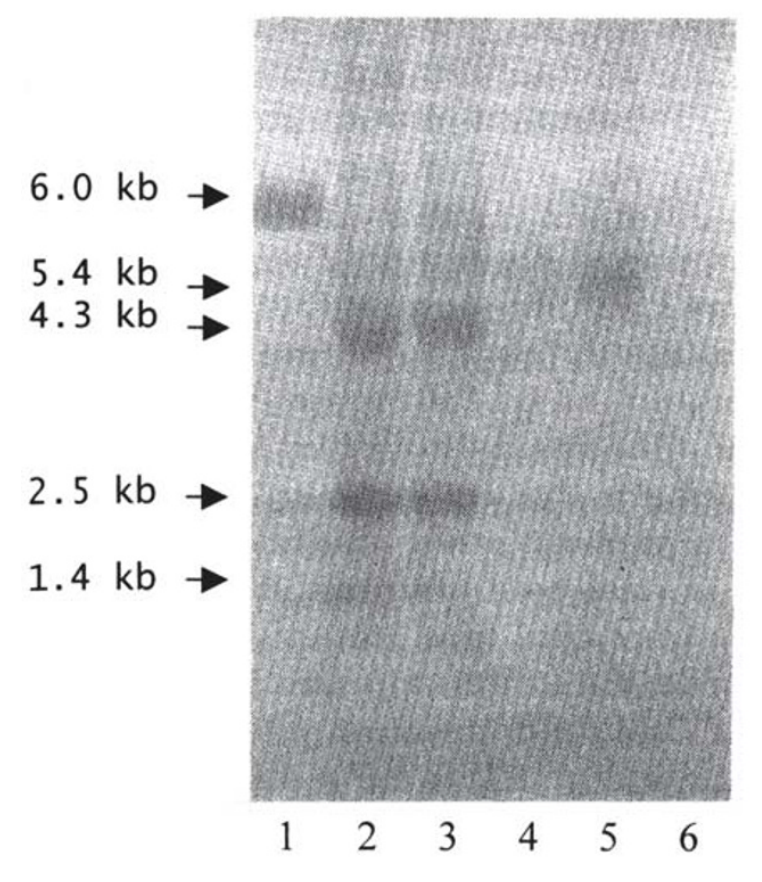

\section{DISCUSSION}

Auxin synthesis genes have been intensively studied for the induction of adventitious roots on plant[9-11]. In our previous study, several plant lines transgenic for auxin genes were selected with improved rooting ability from T0 generation of transformed population and the relation between root system and leafy head formation was evidenced [4]. After four generation of self-cross, some transgenic lines maintained the characters of improved rooting ability and showed earliness of leaf folding and head formation.

Head formation is an important morphological and physiological behavior for Chinese cabbage. T5 plants of the transgenic lines D1232 and D1653 showed the phenotype similar to normal plants. However the difference was identified between them in aspects of germination, leaf folding and head formation. In D1232 and D1653, their properties of 
Auxin genes and Leafy head

less abnormally germinated seeds and earliness of leaf folding and heading (Tab 1, 2 and 3 ) seem to be related to higher auxin content in leaves or/and higher sensitivity of plants to auxin. On medium without auxin, intact leaf blade and apical blade halves of transgenic plant line D1653 produced adventitious roots spontaneously whereas these two sections from control plants did not differentiate any roots (Fig 1A). These results indicate that the leaves of transgenic plants contain a higher concentration of auxin than that of normal plants in which the content of auxin may not be enough to induce root formation. On the other hand, more roots on leaf sections of transgenic lines were found on medium with added low level of auxin (Fig 1B), demonstrating that transgenic leaves may be more sensitive to auxin treatment than untransformed leaves. There was evidence showing that the relatively high content and uneven distribution of auxin concentration in Chinese cabbage can improve the character of head formation[3]. The results in this paper offer additional evidence in this regard.

The function of auxin genes in the regulation of leaf head formation could be attributed to several components. In transgenic plants, the root system is improved as described in our previous report[4]. Secondly, plant growth rate can be increased at a resonable content of auxin. Thirdly, the increased endogenous auxin content can play a direct role in the improvement of leaf folding and head formation.

The D1740 plants showed many phenotypes that usually appear at rather high concentration of auxin, such as inhibition of seed germination, redardation of plant growth, extremely wrinkled leaves and failure of head formation. Thus, it is highly probable that D1741 plants have high auxin content due to the over-expression of foreign auxin genes. The phenotype change in other plants with auxin transgenes have also been described. Tobacco transgenic plants with Agrobacterium tumefacience tms 1 gene (homologous to aux1) produced phenotypically normal plants under the control of its own promoter[12], whereas the tms1 gene under the control of CaMV 19S promoter gave petunia and tobacco plants an extremely abnormal morphology[11]. In our present experiment, AUX genes consisted of aux 1 and aux 2 under the control of their own promoters gave Chinese cabbage plants either normal or extremely abnormal morphology.

Three types of T5 transgenic lines could be classified: D1232 and D1653 belong to the type with rapid growth, D1470 is the type with growth inhibition and D1911 is the type with normal growth. In the previous study, four types of root lines and the three groups of to plant lines were designatid[4]. D1232 with a slightly abnormal shoot and fast growth; D1645 (sister line of D1470) with extremely abnormal shoot and inhibitory growth, and D1434 (sister line of D1911) with normal shoot like the control. After five successive sexual generations, the four transgenic lines kept the their original growth properties. D1232 (and D1653) plants grew faster and formed leafy heads earlier even though they had a slightly abnormal shoots at seedling stage of T0 generation; D1470 plants had rather wrinkled leaves and failed to form leafy heads since they had extremely abnormal seedlings just like that in T0 generation. D1911 maintained the same growth properties as that of control. These results indicated that the modified characters caused by exog- 
enous auxin genes can be transferred genetically from the first generation to late generations.

The beginning of leafy head formation is indicated by the appearance of folding leaves which are the most sensitive to auxin treatment in the experiment for induction of inward-bending leaves[3]. Therefore, the folding leaves were tested for initiation of adventitious roots on leaf cuttings in our experiment. On the medium without auxin, the transgenic leaf sections produced adventitious roots spontaneously while the control sections did not, indicating that the former contained the higher level of auxin. On the medium with low level of auxin, much more roots were found on transgenic leaf sections than on control sections. Apparently, transgenic tissue was more sensitive to exogenous auxin than the control. On the other hand, three leaf sections were significantly different in induction of adventitious roots in that the cut end of intact leaf produced the most roots while that of blade halves gave the least roots. It is not known whether such gradient root induction on leaf sections was caused by uneven distribution of auxin in leaf that was considered to be related to leaf folding[3]. Recently, $B c p L H$ gene that was preferentially expressed in folding leaf was isolated from Chinese cabbage[13]. It was found that spray of auxin (IAA) on dorsal surface of leaf strongly enhanced the expression of $B c p L H$ gene. Thus, an experiment is designed to evaluate the expression pattern of BcpLH gene in various organs of Chinese cabbage with or without auxin transgenes.

The three types of transgenic lines represent three levels of auxin gene expression and therefore could be used for further study of the comparative evaluation of auxin gene function in differentiation and development of plant organs of Chinese cabbage. Since D1232 and D1653 have the advantage of rapid growth and early head formation, they are good materials for breeding of new variety of Chinese cabbage with rapid growth and earliness of head formation.

\section{ACKNOWLEDGEMENTS}

This project was supported by National Natural Science Foundation of China (Grant No. 39870450).

\section{REFERENCES}

[1] He YK, Yu XH. Pattern and mechanism of morphological transformation of plant organs (in Chinese). In Li CS (ed), Advances in Plant Science (Vol. 1). Higher Education Publisher, Beijing 1998; pp.16479.

[2] Ito H, and Kato T. Studies on the head formation of Chinese cabbage: Histological and physiological studies of head formation. J Jpn Soc Hortic Sci 1957; 26:154-204

[3] Ito H, Kato T. Studies on the head formation of Chinese cabbage. II. Relation between auxin and head formation (in Japanese). Agric Hortic 1958; 26:771.

[4] He YK, Wang JY, Gong ZH et al. Root development initiated by exogenous auxin genes in Brassica ssp. Plant Physiol Biochem 1994; 32(4):493-500.

[5] Camilleri C, Jouanin L. The Tr-DNA region carrying the auxin synthesis genes of the Agrobacterium rhizogenes agropine-type plasmid pRiA4: Nucleotide sequence analysis and introduction into tobacco 


\section{Auxin genes and Leafy head}

plants. Mol Plant-Microbe Interaction 1991; 4:155-62.

[6] Offringa I, Melchers L, Regensburg-Tuink A et al. Complementation of Agrobacterium tumefaciens tumor-inducing aux mutants by genes from the TR-region of Ri plasmid of Agrobacterium rhizogenes. Proc Natl Acad Sci USA 1986; 83:6935-9.

[7] Inze D, Follin A, van Lijsebettens $\mathrm{M}$ et al. Genetic analysis of individual T-DNA genes of Agrobacterium tumefaciens; further evidence that two genes are involved in indole-3-acetic acid synthesis. Mol Gen Genet 1984; 194:265-74.

[8] Sambrook J, Fritsch EF, Maniatis T. Molecular cloning: A laboratory mannual. Cold Spring Harbor. NY, 1989.

[9] Cardarelli M, Spano L, Mariotti D et al. The role of auxin in hairy root induction. Mol Gen Genet 1987; 208:457-63.

[10]\} Cardarelli M, Mariotti D, Pomponi M et al. Agrobacterium rhizogenes T-DNA genes capable of inducing root phenotype. Mol Gen Genet 1987; 209:475-80.

[11] Klee H, Horsch R, Hinchee M et al. The effects of overproduction of two Agrobacterium tumefaciens T-DNA auxin synthetic gene products in transgenic petunia plants. Genes Dev 1987; 1:86-96.

[12] Follin A, Inze D, Budar F et al. Genetic evidence that the tryptophan 2-mono-oxygenase gene of Pseudimonas savastanoi is functioally equivalent to one of the T-DNA genes involved in plant tumour formation by Agrobacterium tumefacience. Mol Gen Genet 1985; 201:178-85.

[13] Yu XH, Peng JS, Feng XZ, Yang SX, ZHeng ZR, Tang XR, Shen RJ, Liu PL, He YK. Cloning and structural and expressional characterization of BcpLH gene preferentially expressed in folding leaf of Chinese cabbage. Science in China (Series C) 2000; 43:321-9.

Received April-24-2000. Revised May-29-2000. Accepted May-30-2000. 